\title{
Twin boundary spacing-dependent friction in nanotwinned copper
}

\author{
Junjie Zhang, ${ }^{1}$ Yujie Wei, ${ }^{2, *}$ Tao Sun, ${ }^{1, \dagger}$ Alexander Hartmaier, ${ }^{3}$ Yongda Yan, ${ }^{1}$ and Xiaodong $\mathrm{Li}^{4}$ \\ ${ }^{1}$ Center for Precision Engineering, Harbin Insitute of Technology, Harbin 150001, China \\ ${ }^{2}$ State Key Laboratory of Nonlinear Mechanics, Institute of Mechanics, Chinese Academy of Sciences, Beijing 100190, China \\ ${ }^{3}$ Interdisciplinary Centre for Advanced Materials Simulation (ICAMS), Ruhr University Bochum, Stiepeler Strasse 129 (UHW), \\ D-44801 Bochum, Germany \\ ${ }^{4}$ Department of Mechanical Engineering, University of South Carolina, 300 Main Street, Columbia, South Carolina 29208, USA
}

(Received 28 July 2011; published 13 February 2012)

\begin{abstract}
The deformation mechanisms of nanotwinned $\mathrm{Cu}$ subjected to nanoscratching are investigated by means of molecular dynamics simulations. Scratching simulations on nanotwinned single-crystalline $\mathrm{Cu}$ with the twin planes parallel and perpendicular to the scratching direction are performed. Since the detwinning mechanism is completely suppressed in the former case, no apparent correlation between frictional coefficient and the twin spacing is observed. In samples where the twin planes are perpendicular to the scratching direction, the friction increases as the twin spacing decreases, and then decreases as the twin spacings become even smaller. It results from the competitive plastic deformation between the inclined dislocations and the detwinning mechanism. Subsequent simulations for nanotwinned polycrystalline $\mathrm{Cu}$ unveil that in addition to the grainboundary-associated deformation mechanism, dislocation-mediated detwinning plays a significant role in the plastic deformation of nanotwinned $\mathrm{Cu}$. The twin boundary spacing in turn affects nanotwinned materials to resist scratching via plastic deformation. We demonstrate via the nanoscratching tests that there exists a critical twin boundary spacing for which the friction coefficient is maximized and that this transition results from the competing deformation mechanisms in those nanotwinned materials.
\end{abstract}

DOI: 10.1103/PhysRevB.85.054109

PACS number(s): 62.20.Qp, 62.20.F-, 62.25.-g

\section{INTRODUCTION}

Nanotwinned (nt) $\mathrm{Cu}$ containing a high density of growth twin boundaries (TBs) has drawn a wide range of interest due to its high strength, intermediate ductility, and high electric conductivity in comparison with its twin-free counterpart. ${ }^{1,2}$ There is a compelling need to understand the atomic origin of the unique mechanical properties of nt $\mathrm{Cu}$. Both experimental and theoretical efforts have been made in investigating the deformation behavior of $\mathrm{nt} \mathrm{Cu}$ subjected to different loading modes, including tension, rolling, and nanoindentation. ${ }^{3-12} \mathrm{It}$ is found that the high strength of $\mathrm{nt} \mathrm{Cu}$ is a result of TBs acting as effective obstacles to dislocation motion, while the eminent ductility is owing to the enhanced dislocation nucleation sites provided by TBs. To date, limited information is known about the deformation mechanism of $\mathrm{nt} \mathrm{Cu}$ during wear process. For its promising applications as functional structures, a fundamental understanding of the deformation mechanism of $\mathrm{nt} \mathrm{Cu}$ during wear is greatly needed. The purpose of this work is to unveil the origin of wear for $\mathrm{nt} \mathrm{Cu}$ at the nanoscale, which is expected to advance our understanding of the atomic nature of tribological behavior of nanostructured materials. ${ }^{13-18}$

Recent experimental tests and atomistic simulations have shown that the strength of $\mathrm{nt} \mathrm{Cu}$ has a strong dependence on twin boundary spacing (TBS). ${ }^{19-21}$ The strength of $\mathrm{nt} \mathrm{Cu}$ first increases with decreasing TBS in a Hall-Petch manner. However, after reaching the maximum strength at the critical TBS, strength softening occurs when TBS further decreases. ${ }^{19}$ In addition, it is found that the critical TBS depends on grain size; i.e., the smaller the grain size, the smaller the critical twin thickness. ${ }^{12,22}$ Therefore, it is of great interest to study the effect of TBS on the wear of nt $\mathrm{Cu}$.

In this study, we elucidate the TBS-dependent deformation mechanisms that work in $\mathrm{nt} \mathrm{Cu}$ subjected to nanoscratching using molecular dynamics (MD) simulations. We start with rather simple nanotwinned single-crystalline $\mathrm{Cu}$ with twin planes either perpendicular or parallel to the surface. Our simulations suggest that both the TBS and the inclined angle between the surface and twin planes strongly affect the operating deformation modes. More practical nanotwinned polycrystalline $\mathrm{Cu}$ is then simulated, where dislocations inclined to twin planes, dislocation-accommodated detwinning, and grain boundary (GB) associated mechanisms start to interplay with each other. Based on those investigations, we unveil that TBS has a strong influence on the deformation mechanism and resulting surface morphology of nt $\mathrm{Cu}$. A critical TBS for the maximum friction coefficient is discovered. This "optimal" frictional behavior results from the plastic deformation transition. ${ }^{12,22,23}$

\section{SIMULATION METHOD}

Preparations for nanotwinned single-crystalline $\mathrm{Cu}$ samples are relatively straightforward. Two particular twin plane orientations are considered: The twin planes are perpendicular to the scratching direction in one set, and those in the other set are parallel to the scratching direction. For each set of nt single-crystalline $\mathrm{Cu}$, six samples with different twin spacing are considered. The twin spacings are $0.63 \mathrm{~nm}, 1.25 \mathrm{~nm}$, $1.88 \mathrm{~nm}, 2.50 \mathrm{~nm}, 3.10 \mathrm{~nm}$, and $3.75 \mathrm{~nm}$. For nt polycrystalline $\mathrm{Cu}$, five fully three-dimensional samples composed of nanoscale TBs with different TBS embedded in grains, from $0.63 \mathrm{~nm}, 1.25 \mathrm{~nm}, 1.67 \mathrm{~nm}, 2.92 \mathrm{~nm}$, to $3.75 \mathrm{~nm}$, are prepared by using the Voronoi construction. The grain structure for each nt $\mathrm{Cu}$ sample is the same, and each sample contains 12 grains with an average grain size of $10 \mathrm{~nm}$, and correspondingly the atomic structure of the five samples is shown in Fig. 1. The dimension for each $\mathrm{nt} \mathrm{Cu}$ sample is $30 \mathrm{~nm}$ by $10 \mathrm{~nm}$ by 

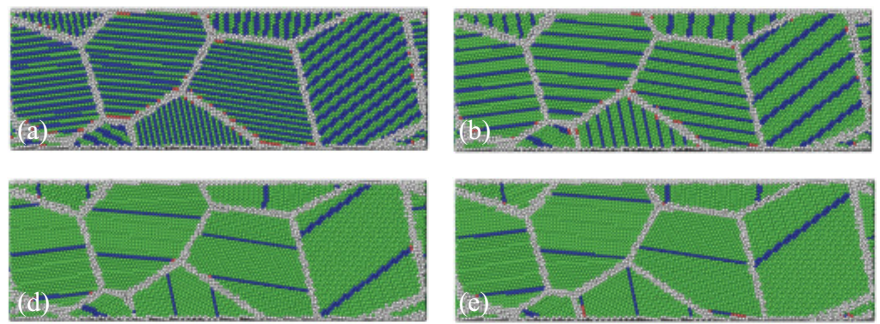

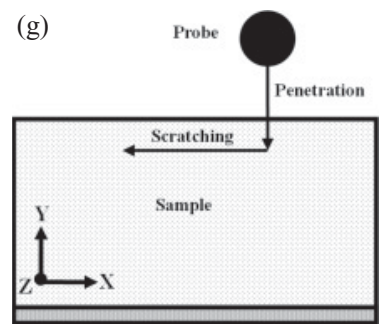

FIG. 1. (Color online) Atomic configurations of (a)-(e) nt $\mathrm{Cu}$ and (f) single-crystalline $\mathrm{Cu}$ samples. The TBS for each nt $\mathrm{Cu}$ sample is (a) spacing 1 of $0.63 \mathrm{~nm}$, (b) spacing 2 of $1.25 \mathrm{~nm}$, (c) spacing 3 of $1.67 \mathrm{~nm}$, (d) spacing 4 of $2.92 \mathrm{~nm}$, and (e) spacing 5 of $3.75 \mathrm{~nm}$. Blue, green, and gray stand for TB atoms, fcc atoms, and surface and GB atoms, respectively. (g) Illustration for the scratching process.

$30 \mathrm{~nm}$ in the $X, Y$, and $Z$ directions, respectively. The atomic interactions between face-centered-cubic (fcc) $\mathrm{Cu}$ atoms are described by the embedded atom method potential. ${ }^{24}$ Periodic boundary conditions are applied in the $X$ and $Z$ directions. Prior to nanoscratching, the as-created nt $\mathrm{Cu}$ samples are first relaxed to their equilibrium configurations by the following procedure: The atoms in the samples are first relaxed to their minimum energy configurations using the FIRE (fast inertia relaxation engine) algorithm..$^{25}$ Then the samples are heated up to $30 \mathrm{~K}$ by dynamic MD simulation using the Nose-Hoover thermostat for $40 \mathrm{ps}$ in the isothermal-isobaric NPT ensemble.

The equilibrated $\mathrm{Cu}$ samples are then subjected to nanoscratching using a spherical diamond probe with a radius of $4 \mathrm{~nm}$ in the microcanonical $N V E$ ensemble. The interaction between the probe and the samples is represented by a strong repulsive potential. ${ }^{26}$ During nanoscratching, the probe first penetrates into the $\mathrm{Cu}$ sample by $1.39 \mathrm{~nm}$ along the negative $Y$ direction at a constant velocity of $20 \mathrm{~m} / \mathrm{s}$, and then scratches $12.22 \mathrm{~nm}$ along the negative $X$ direction at a constant velocity of $20 \mathrm{~m} / \mathrm{s}$. Figure $1(\mathrm{~g})$ illustrates the scratching process.

All MD simulations are completed using the classical molecular dynamics package IMD with a time step of $1 \mathrm{fs} .^{27}$ The software OVITO is utilized to visualize MD data and generate MD snapshots. ${ }^{28}$ The common neighbor analysis (CNA) is adopted to identify the feature of each atom before and after deformation during nanoscratching, and the difference between intrinsic stacking fault (ISF) and TB is further distinguished..$^{29,30}$ In this study, atoms are colored based on the following protocol: Green stands for fcc atoms, red for ISF atoms, blue for TBs atoms, and gray for other atoms which include surface atoms, GB atoms, and dislocation cores.

\section{SCRATCHING RESPONSE IN nt SINGLE-CRYSTALLINE $\mathrm{Cu}$}

We start with understanding rather simple nanotwinned single-crystalline $\mathrm{Cu}$ where twin planes are either parallel or perpendicular to the scratching direction. For the case of parallel twins (with respect to the scratching direction), there is no apparent change in the frictional coefficient as the twin spacings decrease from $3.75 \mathrm{~nm}$ to $0.63 \mathrm{~nm}$ [Fig. 2(a)]. By looking at the detailed deformation process, we see that plastic deformation in these samples is dominated by dislocations nucleated from the free surface and inclined to twin planes [Figs. 2(b) to 2(g)]. The existence of twin boundaries only influences those dislocations which pass through the twin boundaries, and the fraction of such dislocations is small. This deformation mechanism leads to almost no change in the frictional coefficient as the twin spacings decrease. As a comparison, scratching in nanotwinned single-crystalline copper with perpendicular twin planes (with respect to scratching direction) is also investigated. The frictional coefficient first increases as the twin spacing decreases, and then decreases as twin spacings become even smaller [Fig. 2(h)]. From the deformation snapshots given in Figs. 2(i) to 2(n), we see that there exist two types of competitive dislocation activities, with one being inclined to the twin boundaries and the other parallel to the twin boundaries. The competition of these two mechanisms resembles what has been seen in nanotwinned polycrystalline metals, ${ }^{12,22,23}$ which gives rise to a maximum friction coefficient at a certain twin spacing seen in Fig. 2(h).

\section{FRICTIONAL BEHAVIOR IN nt POLYCRYSTALline Cu}

With the understanding we developed in Sec. III in nanotwinned single-crystalline $\mathrm{Cu}$, we analyze more practical nanotwinned polycrystalline $\mathrm{Cu}$ here. Figures 3(a) and 3(b) show the cross-sectional views of spacing 5 before and after penetration, respectively. For all nt $\mathrm{Cu}$ samples studied the penetration positions are the same, at the center surface of the grain indicated by white arrow in Fig. 3(a). The $\mathrm{Cu}$ sample undergoes elastic deformation during the initial stage of penetration, followed by plastic deformation through the nucleation of dislocations from the penetrated surface. It is seen from Fig. 3(b) that dislocation motion is blocked by TBs and GBs, and the TB beneath the probe is dislocated by the penetration of a nucleated dislocation inclined to the twin plane. Simultaneously, the grain structure beneath the probe undergoes a significant change. The GB on the right side of penetration region is deformed remarkably, as shown in Fig. 3(b). In addition, dislocation intersecting with TBs in neighboring grains is also observed. The detailed description of deformation of $\mathrm{nt} \mathrm{Cu}$ during penetration can also be found in Ref. 10.

In nanoscratching the force acting on the probe is composed of three components: scratching force along the $X$ direction, normal force along the $Y$ direction, and lateral force along the $Z$ direction. We follow the normal definition of friction coefficient as the ratio of scratching force to normal force. Figure 4(a) shows the friction coefficient versus scratching length curves obtained from nanoscratching on respective 

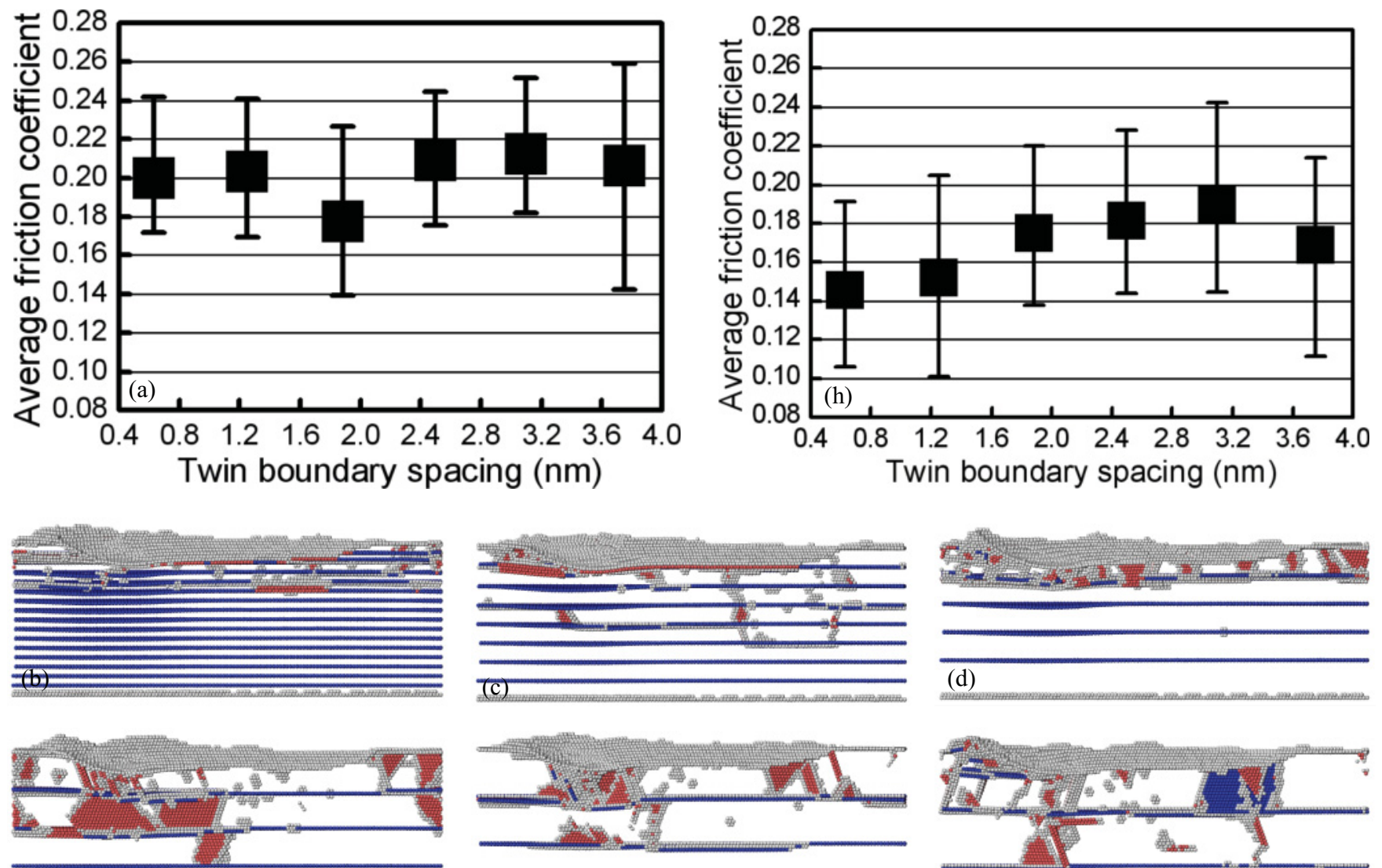

(e)
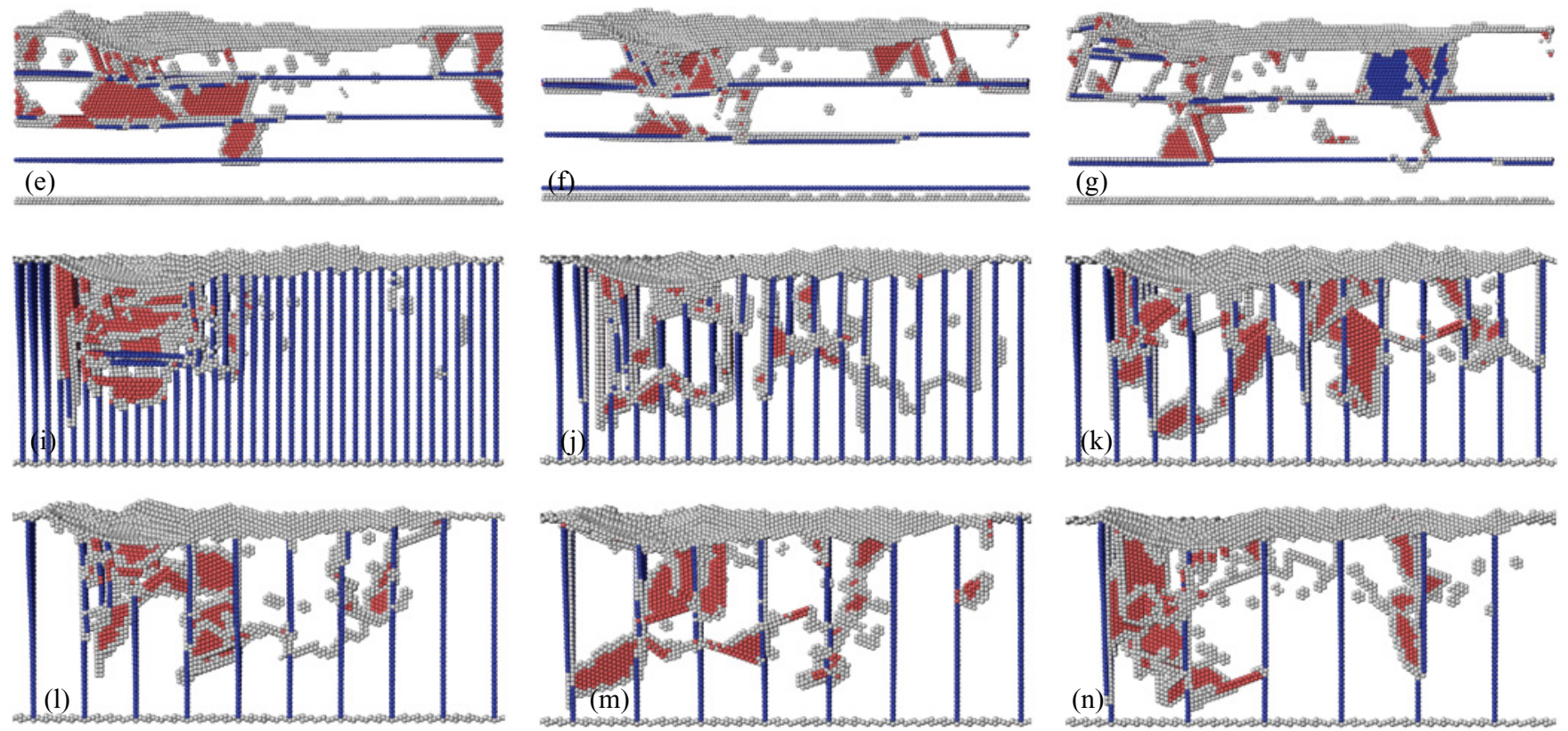

FIG. 2. (Color online) Frictional behavior in nt single-crystalline Cu samples with different TBS. (a) Dependence of frictional coefficient and $[(\mathrm{b})-(\mathrm{g})]$ deformation snapshots on twin spacing for $\mathrm{nt}$ single-crystalline $\mathrm{Cu}$ where twin planes are parallel to the scratching direction. (h) Dependence of frictional coefficient and [(i)-(n)] deformation snapshots on twin spacing for nt single-crystalline Cu where twin planes are perpendicular to the scratching direction. Perfect fcc atoms are eliminated in (b)-(g) and (i)-(n) to show defect structures clearly.

nt $\mathrm{Cu}$ and single-crystal $\mathrm{Cu}$ samples. The scratching route illustrated in Fig. 4(b) involves three grains and crosses two $\mathrm{GBs}$, which is the same for each nt $\mathrm{Cu}$ sample studied. The curves show common features: Frictional resistance increases rapidly at the beginning stage of nanoscratching, and then fluctuates strongly about a constant average value when scratching is stable. In addition, Fig. 4(a) demonstrates that the friction coefficient increases to a local high value when the bottom of probe is approaching GBs for all nt $\mathrm{Cu}$ samples studied. Figure 4(c) shows the constant average values of the friction coefficient between scratching lengths of $4.70 \mathrm{~nm}$ and $12.22 \mathrm{~nm}$ for $\mathrm{nt} \mathrm{Cu}$ samples. The error bars present the lowest and highest values within the same range of scratching length for each nt $\mathrm{Cu}$ sample. The latter stage of fluctuation in the frictional coefficient is attributed to the inhomogeneous nature of plastic deformation during nanoscale scratching, and the constant average value depends strongly on TBS: It first increases with increasing TBS, and reaches the maximum for 


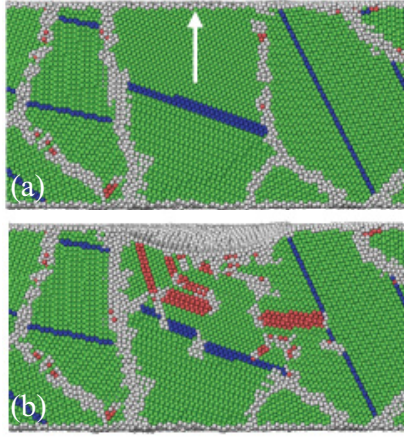

FIG. 3. (Color online) Cross-sectional views of spacing 5 (a) before penetration and (b) after penetration. The white arrow in (a) indicates the penetration position.

spacing 4 with a TBS of $2.92 \mathrm{~nm}$, then decreases with further increase in TBS. This indicates that there exists a critical TBS of $2.92 \mathrm{~nm}$ for which the constant average value of friction coefficient is maximized. We note, however, that this might be only a local maximum, since the friction coefficient for the single-crystalline $\mathrm{Cu}$ is the largest of all; i.e., for very large TBS another increase in the friction coefficient has to be expected, due to a change in the deformation mechanism, because single-crystalline $\mathrm{Cu}$ deforms exclusively

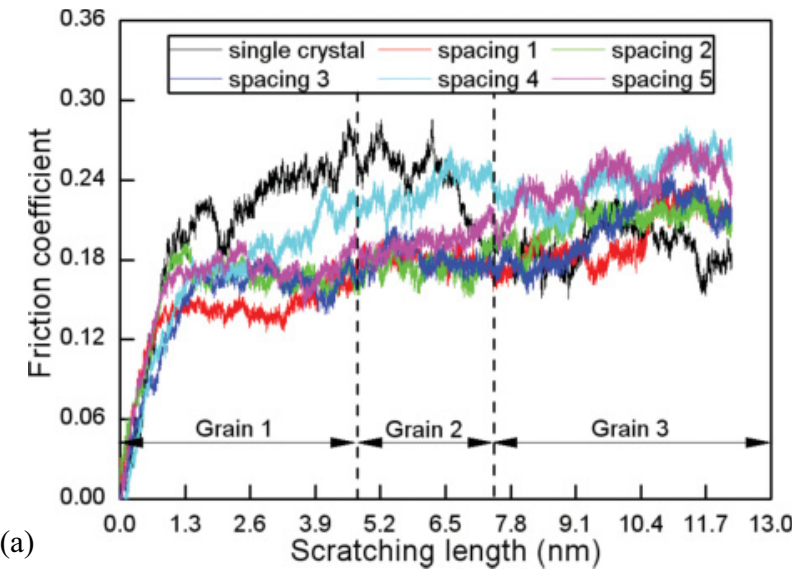

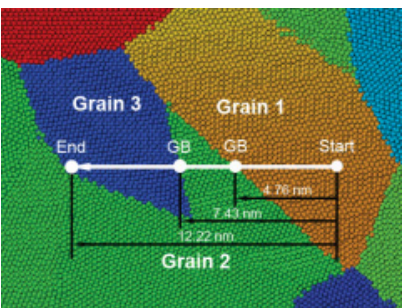

(b)

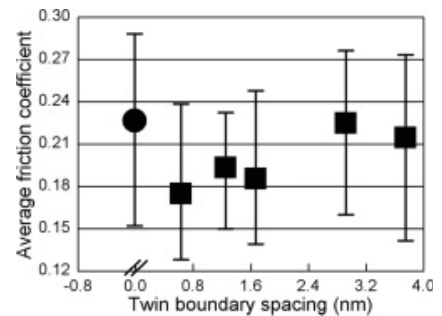

(c)
FIG. 4. (Color online) Nanoscratching on nt $\mathrm{Cu}$ samples with different TBS. (a) Friction coefficient-scratching length curves. (b) Illustration of scratching route. Atoms in different grains are colored differently. (c) Variations in average friction coefficient with TBS, and error bars present the lowest and highest values within the same range of scratching length between $4.70 \mathrm{~nm}$ and 12.22 $\mathrm{nm}$. The average friction coefficients of single-crystalline and $\mathrm{nt}$ $\mathrm{Cu}$ samples are presented by using the circle and square symbols, respectively.
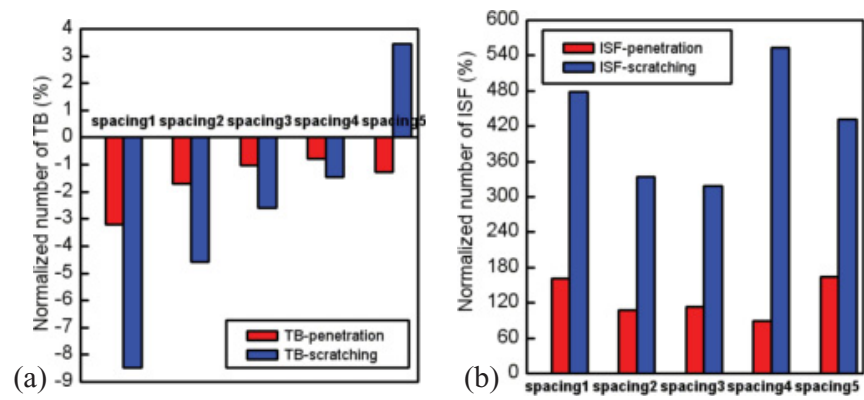

FIG. 5. (Color online) Variations in normalized numbers of (a) TB and (b) ISF atoms at different periods during nanoscratching on $\mathrm{nt} \mathrm{Cu}$ samples. The normalized number is defined as the ratio between the change in number and the initial number of different atom types.

by dislocation motion. Furthermore, we have to expect that the exact value of the critical TBS depends on the diameter of the indenter and also on the indentation depth.

Figures 5(a) and 5(b) plot the variations in normalized numbers of TB and ISF atoms at different periods during nanoscratching on nt $\mathrm{Cu}$ samples, respectively. It is seen that when TBS is below or equal to the critical value, the number of TB atoms decreases accompanied with an increase in the number of ISF atoms during nanoscratching. In addition, the decrease in the number of TB atoms is more pronounced for smaller TBS. In contrast, the number of TB atoms is increased after nanoscratching on spacing 5, the TBS of which is larger than the critical value. This suggests that the critical TBS for the maximum constant average value of friction coefficient is contributed by the strong competition of twinning migration (dislocations residing in twin planes) and nucleation of isolated dislocations either parallel or inclined to twin planes. Specifically, nucleation of isolated dislocations plays a more pronounced role in the plastic deformation of $\mathrm{nt}$ $\mathrm{Cu}$ when TBS is larger than the critical value.

Figure 6 shows the cross-sectional views of various $\mathrm{Cu}$ samples after nanoscratching, demonstrating that TBS has a significant influence on the deformation mechanism of $\mathrm{nt}$ $\mathrm{Cu}$ samples. It is seen that in the nt $\mathrm{Cu}$ samples with larger TBS, dislocation density is smaller, while evolution of grain structure beneath the scratched surface is more pronounced. Figures 6(a) to 6(c) demonstrate that partial dislocations mainly glide parallel to TBs - twin boundary migration, which is evident by checking the reduced twin boundaries in the middle of the two grains in those snapshots. Widening of TBS in the neighboring grain is highlighted by the white arrow in comparison with other grains. However, nucleation of isolated partial dislocations either parallel or inclined to twin planes is dominant when TBS is larger, as shown in Figs. 6(d) and 6(e). Figure 6(e) also demonstrates the formation of mechanical TBs, highlighted by white arrow. This demonstrates that the influence of TBS on dislocation activities shown in $\mathrm{nt}$ single-crystalline $\mathrm{Cu}$ is also effective with the presence of GBs.

Figure 7(a) shows the evolution of different kinds of atoms with scratching distance during nanoscratching on spacing 5. The reduced number is calculated by subtracting the base number before scratching using the measured number. 

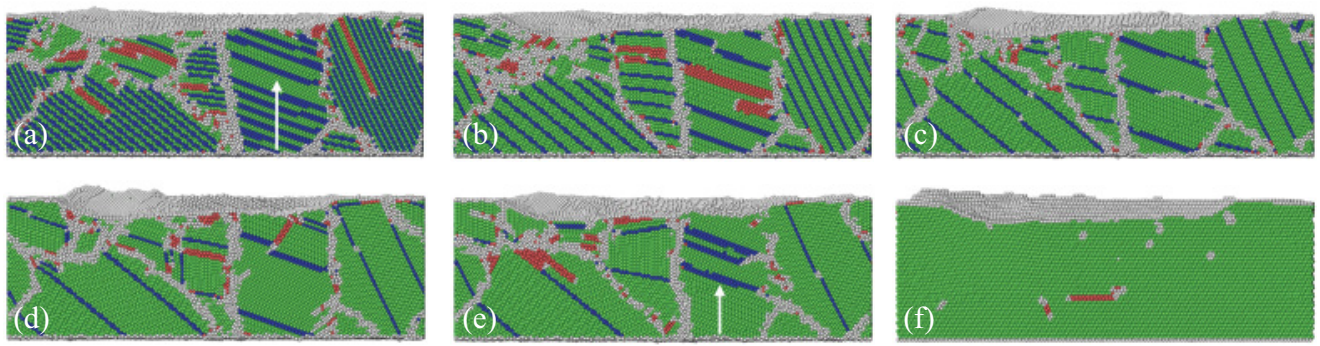

FIG. 6. (Color online) Cross-sectional views of $\mathrm{Cu}$ samples after nanoscratching. (a) Spacing 1, (b) spacing 2, (c) spacing 3, (d) spacing 4, (e) spacing 5, and (f) single crystalline. The white arrows in (a) and (e) highlight the widening of TBS and formation of mechanical TB, respectively.

Figure 7(a) shows that individual deformation mechanisms such as the dislocation mechanism, GB-associated mechanism, deformation twinning, and TB-associated mechanism work in parallel in the plastic deformation of the nt $\mathrm{Cu}$ sample during nanoscratching. In zone I, an increase in fcc atoms accompanied with a decrease in ISF atoms indicates that plastic deformation is dominated by annihilation of partial dislocations. Figure 7(b) shows a dislocation inclined to the TB in zone I. The TB loses its coherency accompanied by the formation of twinning partial dislocation. In contrast, plastic deformation in zone II is dominated by dislocation nucleation and subsequent propagation, as ISF atoms and other atoms increase monotonously. Figure 7(c) shows that a partial dislocation glides parallel to the TB after being nucleated from the GB-TB intersection. In zone III, a strong competition among several deformation mechanisms is discovered. During the initial period, ISF atoms keep decreasing while TB atoms increase with a larger slope than other atoms, indicating that plastic deformation is dominated by deformation twinning. However, at the period highlighted by the dashed ellipse, an increase in other atoms is accompanied with a decrease in both TB and ISF atoms, suggesting that plastic deformation is mediated dominantly by the GB-associated mechanism. In zone IV, although the conspicuous increase in ISF atoms indicates that the dislocation mechanism is one major deformation mechanism, the unchanged number of other atoms reveals that the GB-associated mechanism is another mediating deformation mode, which has strong competition with the dislocation mechanism.

Figure 8 shows the resulting surface morphologies of various $\mathrm{Cu}$ samples after nanoscratching. Atoms are colored according to atomic height. It is seen that the single-crystalline $\mathrm{Cu}$ sample possesses a larger volume of surface pileup than nt $\mathrm{Cu}$ samples. For nt $\mathrm{Cu}$ samples, surface pileup is more pronounced with a larger TBS. It is known that surface pileup results from the accumulation of surface steps formed when the motion of dislocation gliding on $\langle 1-10\rangle\{111\}$ slip systems terminates at the surface ${ }^{31}$ and that the pileup formation is more pronounced in materials with less work hardening. From the MD simulation we conclude that the stronger work hardening in the nt materials is caused by frequent dislocation-twin boundary interactions. While dislocation nucleates and glides freely in a single-crystalline $\mathrm{Cu}$ sample, dislocation nucleation and motion are effectively suppressed by planar defects with nanometer size in $\mathrm{nt} \mathrm{Cu}$ samples. Although dislocation density is larger for the nt $\mathrm{Cu}$ sample with smaller TBS, there are less active slip systems as dislocations mainly glide parallel to TBs. However, there is a larger space for dislocation to multiply in the $\mathrm{nt} \mathrm{Cu}$ sample with a larger TBS, i.e., spacing 5. In addition, the mediating deformation twinning also enriches the number of activate slip systems. ${ }^{32}$ Figure 8 also demonstrates that TBS has a strong influence on the distribution of surface pileup for
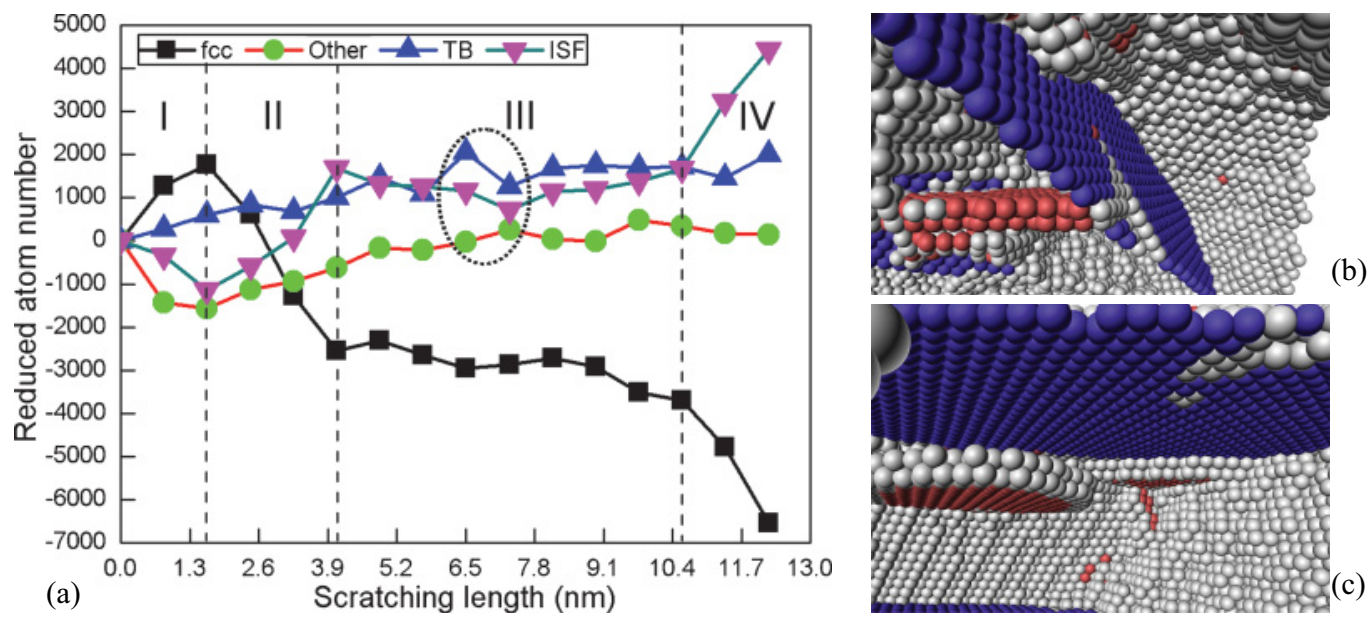

FIG. 7. (Color online) Nanoscratching on spacing 5. (a) Variations of reduced atom number; (b) dislocation inclined to TB; (c) dislocation parallel to TB. 

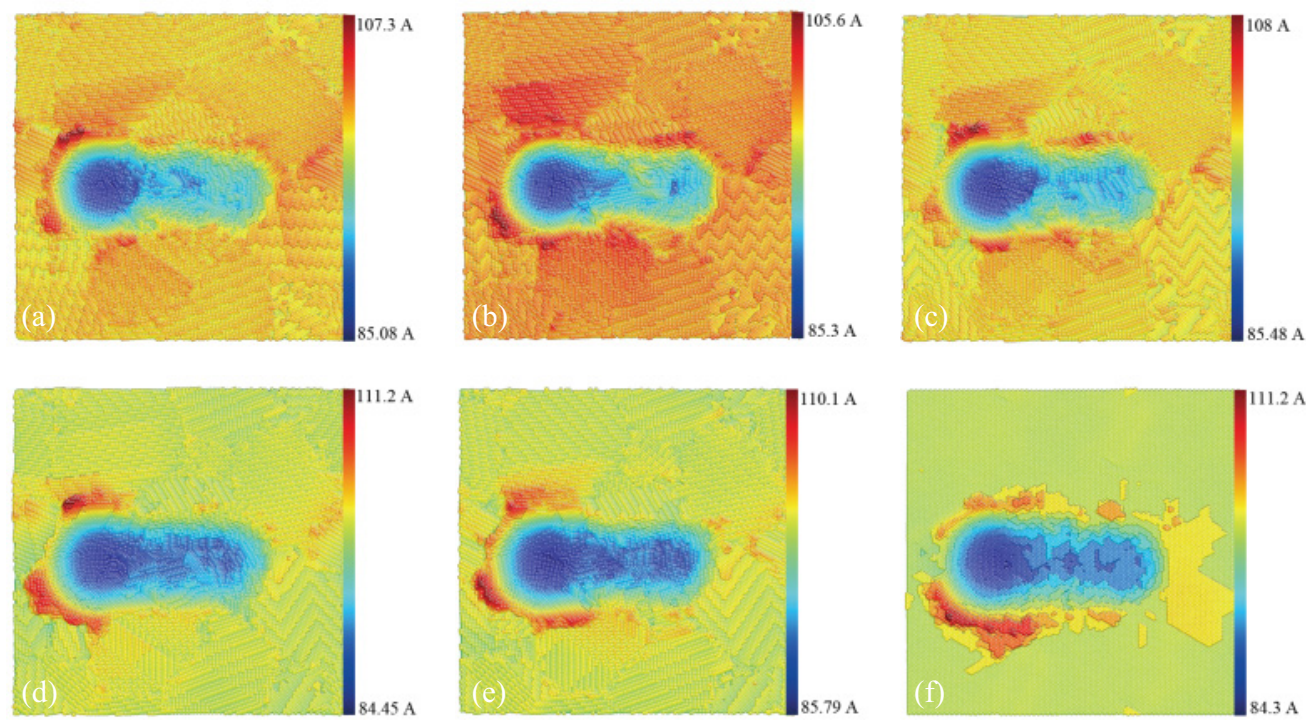

FIG. 8. (Color online) Surface morphologies of various Cu samples after nanoscratching. (a) Spacing 1, (b) spacing 2, (c) spacing 3, (d) spacing 4, (e) spacing 5, and (f) single-crystalline $\mathrm{Cu}$. Atoms are colored according to atomic height.

nt $\mathrm{Cu}$ samples due to different directions of slip systems cutting the surface. While surface pileup events are mainly distributed on both sides of scratched groove when TBS is below or equal to the critical value, pileup locates in the front of groove for spacing 5 where TBS is larger than the critical value.

\section{SUMMARY}

We performed molecular dynamics simulations to study the effect of the twin boundary spacing on nanoscratching in nanotwinned $\mathrm{Cu}$. Our scratching simulations on nanotwinned single-crystalline $\mathrm{Cu}$ with the twin planes parallel and perpendicular to the scratching direction show striking differences in both frictional coefficients and deformation behavior. In the former, we see no apparent correlation between the frictional coefficient and the twin spacing. In the samples where the twin planes are perpendicular to the scratching direction, the friction coefficient increases as the twin spacing decreases, and then decreases as the twin spacings become even smaller. Those differences originate from the competitive plastic deformation between the inclined dislocations and the detwinning mechanism. Subsequent simulation results of $\mathrm{nt} \mathrm{Cu}$ suggest that the dislocation mechanism, GB-associated mechanism, deformation twinning, and TB-associated mechanism work in parallel in the plastic deformation of the $\mathrm{nt} \mathrm{Cu}$ sample during nanoscratching. The TBS has a strong influence on the nanoscratching resistance of $\mathrm{nt} \mathrm{Cu}$. There exists a critical TBS, at which the friction coefficient is maximized, resulting from the strong competition between the two deformation modes. Isolated dislocations either parallel or inclined to twin planes play a more pronounced role in the plastic deformation of $\mathrm{nt}$ $\mathrm{Cu}$ with TBS above the critical value, while twin migration dominates in the samples with smaller TBS. We further observed that the smaller the TBS, the less the volume of surface pileup, which can be attributed to a stronger work hardening in the nt materials with smaller TBS. It shall be noted, however, that the friction coefficient of singlecrystalline $\mathrm{Cu}$ that deforms purely by dislocation-mediated plasticity is higher than that of the nt $\mathrm{Cu}$ samples.

\section{ACKNOWLEDGMENTS}

J.Z., T.S., and Y.Y. acknowledge the financial support from NSFC (50575058 and 50605012). J.Z. thanks A. Stukowski for his valuable discussion and guidance, and ICAMS for providing computational resources. Y.W. acknowledges support from the 973 Project (2011CB711103) and CAS (KJCX2-EW-L03). A.H. acknowledges financial support through ThyssenKrupp AG, Bayer MaterialScience AG, Salzgitter Mannesmann Forschung GmbH, Robert Bosch GmbH, Benteler Stahl/Rohr $\mathrm{GmbH}$, Bayer Technology Services $\mathrm{GmbH}$, and the state of North Rhine-Westphalia, as well as the European Comission in the framework of the European Regional Development Fund (ERDF).

\footnotetext{
*yujie_wei@lnm.imech.ac.cn

†spm@hit.edu.cn

${ }^{1}$ L. Lu, Y. F. Shen, X. H. Chen, L. H. Qian, and K. Lu, Science 304, 422 (2004).

${ }^{2}$ M. Dao, L. Lu, Y. F. Shen, and S. Suresh, Acta Mater. 54, 5421 (2006).

${ }^{3}$ E. Ma, Y. M. Wang, Q. H. Lu, M. L. Sui, L. Lu, and K. Lu, Appl. Phys. Lett. 85, 4932 (2004).
}

${ }^{4}$ Z. X. Wu, Y. W. Zhang, and D. J. Srolovitz, Acta Mater. 57, 4508 (2009).

${ }^{5}$ A. J. Cao and Y. G. Wei, J. Appl. Phys. 102, 083511 (2007).

${ }^{6}$ A. Stukowski, K. Albe, and D. Farkas, Phys. Rev B 82, 224103 (2010).

${ }^{7}$ O. Anderoglu, A. Misra, J. Wang, R. G. Hoagland, J. P. Hirth, and X. Zhang, Int. J. Plasticity 26, 875 (2010). 
${ }^{8}$ L. Lu, R. Schwaiger, Z. W. Shan, M. Dao, K. Lu, and S. Suresh, Acta Mater. 53, 2169 (2007).

${ }^{9}$ Y. Kulkarni and R. J. Asaro, Acta Mater. 57, 4835 (2009).

${ }^{10}$ S. X. Qu and H. F. Zhou, Nanotechnology 21, 335704 (2010).

${ }^{11}$ T. Tsuru, Y. Kaji, D. Matsunaka, and Y. Shibutani, Phys. Rev. B 82, 024101 (2010).

${ }^{12}$ X. Y. Li, Y. J. Wei, L. Lu, K. Lu, and H. J. Gao, Nature (London) 464, 877 (2010).

${ }^{13}$ J. P. Gao, W. D. Luedtke, D. Gourdon, M. Ruths, J. N. Israelachvili, and U. Landman, J. Phys. Chem. B 108, 3410 (2004).

${ }^{14}$ G. T. Gao, R. J. Cannara, R. W. Carpick, and J. A. Harrison, Langmuir 23, 5394 (2007).

${ }^{15}$ I. Szlufarska, M. Chandross, and R. W. Carpick, J. Phys. D: Appl. Phys. 41, 123001 (2008).

${ }^{16}$ Y. F. Mo, K. T. Turner, and I. Szlufarska, Nature (London) 457, 1116 (2009).

${ }^{17}$ Q. Li, Y. Dong, D. Perez, A. Martini, and R. W. Carpick, Phys. Rev. Lett. 106, 126101 (2011).

${ }^{18}$ E. Gnecco, R. Bennewitz, T. Gyalog, C. Loppacher, M. Bammerlin, E. Meyer, and H. J. Guntherodt, Phys. Rev. Lett. 84, 1172 (2000).

${ }^{19}$ L. Lu, X. Chen, X. Huang, and K. Lu, Science 323, 607 (2009).
${ }^{20}$ I. Shabib and R. E. Miller, Modelling Simul. Mater. Sci. Eng. 17, 055009 (2009).

${ }^{21}$ L. Lu, M. Dao, T. Zhu, and J. Li, Scr. Mater. 60, 1062 (2009).

${ }^{22}$ Y. J. Wei, Mater. Sci. Eng. A 528, 1558 (2011).

${ }^{23}$ Y. J. Wei, Phys. Rev. B 83, 132104 (2011).

${ }^{24}$ Y. Mishin, M. J. Mehl, D. A. Papaconstantopoulos, A. F. Voter, and J. D. Kress, Phys. Rev. B 63, 224106 (2001).

${ }^{25}$ E. Bitzek, P. Koskinen, F. Gähler, M. Moseler, and P. Gumbsch, Phys. Rev. Lett. 97, 170201 (2006).

${ }^{26}$ C. Begau, A. Hartmaier, E. P. George, and G. M. Pharr, Acta Mater. 59, 934 (2011).

${ }^{27}$ J. Stadler, R. Mikulla, and H. R. Trebin, Int. J. Mod. Phys. C 8, 1131 (1997).

${ }^{28}$ A. Stukowski, Modelling Simul. Mater. Sci. Eng. 18, 015012 (2010).

${ }^{29}$ J. D. Honeycutt and H. C. Andersen, J. Phys. Chem. 91, 4950 (1987).

${ }^{30}$ A. Stukowski and K. Albe, Modelling Simul. Mater. Sci. Eng. 18, 025016 (2010).

${ }^{31}$ G. Ziegenhain, H. M. Urbassek, and A. Hartmaier, J. Appl. Phys. 107, 061807 (2010).

${ }^{32}$ J. Narayan and Y. T. Zhu, Appl. Phys. Lett. 92, 151908 (2008). 